\title{
Some Linear Transformations with MATLAB Applications, Especially On Infinite Integrals
}

\author{
D.A.GISMALLA \\ Faculty of Mathematics \& Computer Sciences, \\ Gezira University, \\ Wad Medani, P.O.Box 20, \\ SUDAN
}

\author{
OHAMED H.A.ELHEBER \\ Faculty of Mathematics \& Computer Sciences, \\ Gezira University, \\ Wad Medani, P.O.Box 20, \\ SUDAN
}

\begin{abstract}
In this paper, We have considered, listed and described some linear transformations with their applications to sum infinite series accurately to a reasonable number of decimal places, e.g. at least to 10 decimal places of accuracy for most of the applications We give attentions and especial consideration for evaluating an infinite integral section $\oint 5$. This can be done by many linear transformations that can be found in the literature. However, here, We apply two fundamental Linear transformations called Euler's Transformations in section $\oint 3$. , Euler-Maclaurin Summation Formula in section $\oint 4$ and Levin's transform in section $\oint$ 5.1.The Matlab program written for Euler's Transformation uses the diagonal elements for forward difference table as can be found in the Matlab File ForwadDifrenceTable.m in
\end{abstract} Fig.(6(b) ) or obtained directly from

$$
\Delta^{\mathbf{k}} \mathbf{a}_{\mathbf{0}}=\sum_{\mathbf{m}=\mathbf{0}}^{\mathbf{k}}(-\mathbf{1})^{\mathbf{m}}\left(\begin{array}{l}
\mathbf{k} \\
\mathbf{m}
\end{array}\right) \mathbf{a}_{\mathbf{k}-\mathbf{m}}
$$

as in the Matlab File EulerTransform.m in Fig.( 5 ). This means the Matlab program written for Euler's Transformations uses slightly two different approaches and the reader should distinguish carefully between them. The most attractive in this paper the generalized linear transformations which are called Chebyshev Weights and Salzer's Means in section $\oint 2$. For these two methods some fundamental background and the theory of generalized linear transformations are given to furnished to the reader how to follow the program to understand first the constraints on regularity or irregularity for these two method or similar ones.This means that the convergence of the method can be achieved only if these transformations are regular along any path. The path can be along zigzag ,column or a row. To be confident first that our program will be straight and accurately right ,We have used eqn.(3) and eqn.(4) to ensure regularity along any rows according to Toplitz limit theorem as in Theorem 3. Lastly in section $\oint 5$,We consider the most robot method Levin's transform that have been pointed in many references such as [2] and [12]. Levin's transform have been analyzed and modified in many different ways to suit poorly slowly convergent series as in [ 2].

KEY WORDS: Toeplitz limit. Chebyshev, Salzer and Levin's Transformations. Gaussian Quadtrue.

\section{GENERALIZED TOEPLITZ TRANSFORMATIONS}

The most famous result dealing with the regularity of linear transformations is the Toeplitz limit Theorem 1 that concerns the convergence of transformations of $B_{s}$ in Banach space $B_{s}$ where the $(n+1)$ th member of the transformed sequence is a weighted mean of the first $n+1$ members of the original sequence

$$
\bar{s}_{n}=\sum_{k=0}^{n} \mu_{n k} s_{k}
$$

The theory of this transformation is covered quite adequately in the existing literature (Knopp, 1947 , Hardy , 1956, Petersen , 1966, Peyerimhoff, 1969).

Here, We shall state an abstract version of the theorems that shall examine a convergence considerations for $\bar{s}_{n}$ in Eqn. (1) using Toeplitz Limit Theorem 1 and its Toeplitz Regularity in Theorem 2 as in [5]. Now, let, T $(\mathrm{s})=\bar{s}$ is called a generalized Toeplitz transformation .

Theorem 1 (Toeplitz Limit Thearem).

The sum (1) converges, $n \geq 0$. and $\bar{s} \in B_{c}^{2}$ iff

(i) $\left\|\sum_{j=0}^{k} \mu_{n j}\left(s_{j}\right)\right\| \leq M$ for $\left\|s_{j}\right\| \leq 1$

$$
, \mathbf{j} \geq \mathbf{0} \text {, and } \mathbf{n}, \mathbf{k} \geq \mathbf{0} \text {. }
$$

(ii) $\sum_{k=0}^{\infty} \mu_{n k}(y), n \geq 0$, and $\lim _{n \rightarrow \infty} \sum_{k=0}^{\infty} \mu_{n k}(y)$ exist for $y \in B^{I}$,

(iii) $\lim _{n \rightarrow \infty} \mu_{n k}(y)$ exists for $y \in B^{1}, k \geq 0$.

Theorem 2 (Toeplitz Regularity) .

$T(s)=\bar{s}$ is regular for $B_{c}$ iff 
(i) $\left\|\sum_{j=0}^{k} \mu_{n j}\left(s_{j}\right)\right\| \leq M$ for $\left\|s_{j}\right\| \leq 1$

$$
\text { , j } \geq \mathbf{0}, \mathbf{n}, \mathbf{k} \geq \mathbf{0}
$$

(ii) $\sum_{k=0}^{\infty} \mu_{n k}(y)=y+\sigma(1), n \geq 0, y \in B$;

(iii) $\mu_{n k}(y)=\sigma(1)$ in $n, k \geq 0, y \in \mathrm{B}$.

1.1 Complex Toeplitz Methods

The only possible linear transformation is

$$
\mu_{n k}(z)=\mu_{n k} z, \mu_{n k} \in \mathrm{B}
$$

In all practical situations $\mu_{n k}=0, \mathrm{k}>\mathrm{n}$, and so the matrix

$\mathbf{u}=\left[\mu_{\mathrm{nk}}\right]=\left[\begin{array}{cccc}\mu_{00} & 0 & 0 & \ldots \\ \mu_{10} & \mu_{11} & 0 & \ldots \\ \mu_{20} & \mu_{12} & \mu_{22} & \ldots \\ \vdots & \vdots & \vdots & \ddots\end{array}\right]$

is lower triangular. If rows sum to 1 ,

$$
\sum_{k=0}^{n} \mu_{n k}=1,
$$

then $\mathrm{U}$, or the transformation defined by $\mathrm{U}$,

$$
\begin{aligned}
& \mathrm{T}(\mathrm{S})=\bar{s}, \\
& \overline{\mathbf{s}}_{\mathbf{n}}=\sum_{\mathbf{k}=\mathbf{0}}^{\mathbf{n}} \boldsymbol{\mu}_{\mathbf{n k}} \mathbf{s}_{\mathbf{k}}, \mathbf{n}=\mathbf{0}, \mathbf{1}, \mathbf{2}, \ldots
\end{aligned}
$$

is called a triangle .

We now restate the Toplitz limit theorem in a form suitable for $U$ in Eqn.(6) in Theorem 3

Theorem $3 \mathrm{U}$ is regular iff

(i) $\sum_{k=0}^{n}\left|\mu_{n k}\right| \leq M$;

(ii) $\sum_{k=0}^{n} \mu_{n k}=1+\sigma(1)$;

(iii) $\mu_{n k}=\sigma(1), k$ fixed

For the proof of these three theorems and the given in formations, the reader should see the pages (24-27) in [5]..

\section{GENERALIZED LINEAR TRANSFORMATIONS}

\subsection{The Chebyshev Weights}

What is probably the best of all the positive triangles results when $p_{n}(x)$ of the previous section is chosen to be the Chebyshev polynomial $T_{n}(x)$ of degree $\mathrm{n}$. The efficiency of $U$ in this case is a consequence of the extraordinary interpolatory properties of $T_{n}(x)$ that system of polynomials to play such an important role in numerical analysis and approximation theory.

$$
\mathbf{T}_{\mathbf{n}}(\mathbf{x})=\cos \mathbf{n} \theta,
$$

so $T_{0}=1$

$$
\theta=\arccos x, n \geq 0 \text {. }
$$

$T_{n}(x)=\frac{1}{2}\left[\begin{array}{c}\left(x+\sqrt[i]{1-x^{2}}\right)^{n} \\ +\left(x-\sqrt[i]{1-x^{2}}\right)^{n}\end{array}\right]$

another useful representation is :

$$
\left.T_{n}(\mathrm{x})=2^{\mathrm{F}} 1^{\left(\begin{array}{c}
\mathrm{n},-\mathrm{n} \\
\frac{1}{2}
\end{array}\right.} \frac{1-\mathrm{x}}{2}\right)
$$

letting $\mathrm{x}=\frac{2 \gamma}{\mathrm{a}}+1$ gives a positive

triangle with entries

$\mathrm{u}_{\mathrm{nk}}=\frac{(\mathrm{n})_{\mathrm{k}}}{\left(\frac{1}{2}\right)_{\mathrm{k}} \mathrm{a}^{\mathrm{k}}} \frac{\left(\begin{array}{l}\mathrm{n} \\ \mathrm{k}\end{array}\right)}{\sigma_{n}(a)}$

$\sigma_{n}(a)=\frac{\left[\begin{array}{c}(\sqrt{a+1}+1)^{2 n}+ \\ \sqrt{a+1}-1)^{2 n}\end{array}\right]}{2 a^{\mathrm{n}}}$,

Usually one takes $a=1$. As an example 1 of the power of U take $\mathrm{a}=1$ in Eq. (5) and compute as in

Example 1

$$
\begin{aligned}
& S_{n}=\left(\ln (2)_{\mathrm{n}}=\sum_{\mathrm{k}=0}^{\mathrm{n}} \frac{(-1)^{\mathrm{k}}}{\mathrm{k}+1}\right. \\
& \rightarrow \ln 2=0.69314718
\end{aligned}
$$

The results are in Table 1 accurately to 14 D.Ps. and all the programs combines to do this are ChebyWeights.m and ChebyWeightSeries.m files in Fig.(1) and Fig.(2) respectively.

Table 1 shows error $=$
$\log (2)-\mathbf{0 . 6 9 3 1 4 7 1 8 0 5 5 9 9 5 4 = - 8 . 6 5 9 7 3 9 e - 1 5}$

\begin{tabular}{rc}
\hline $\mathrm{n}$ & su \\
& \\
1 & 1.000000000000000 \\
2 & 0.666666666666667 \\
3 & 0.686274509803922 \\
4 & 0.693602693602694 \\
5 & 0.693125361062970 \\
6 & 0.693150956487264 \\
7 & 0.693146851108180 \\
8 & 0.693147230444046 \\
9 & 0.693147174862919 \\
10 & 0.693147181406835 \\
11 & 0.693147180451170 \\
12 & 0.693147180576273 \\
13 & 0.693147180557710 \\
14 & 0.693147180560286 \\
15 & 0.693147180559898 \\
16 & 0.693147180559954
\end{tabular}

\subsection{Salzer Means}

Salzer means are given by

$\mu_{n k}=(-1)^{n+k} \frac{(\gamma+k)^{n}}{\mathrm{n} !}\left(\begin{array}{l}\mathrm{n} \\ \mathrm{k}\end{array}\right), \gamma>0(10)$ and $\mathrm{U}$ transform is given by Eqn.(6), similarly 


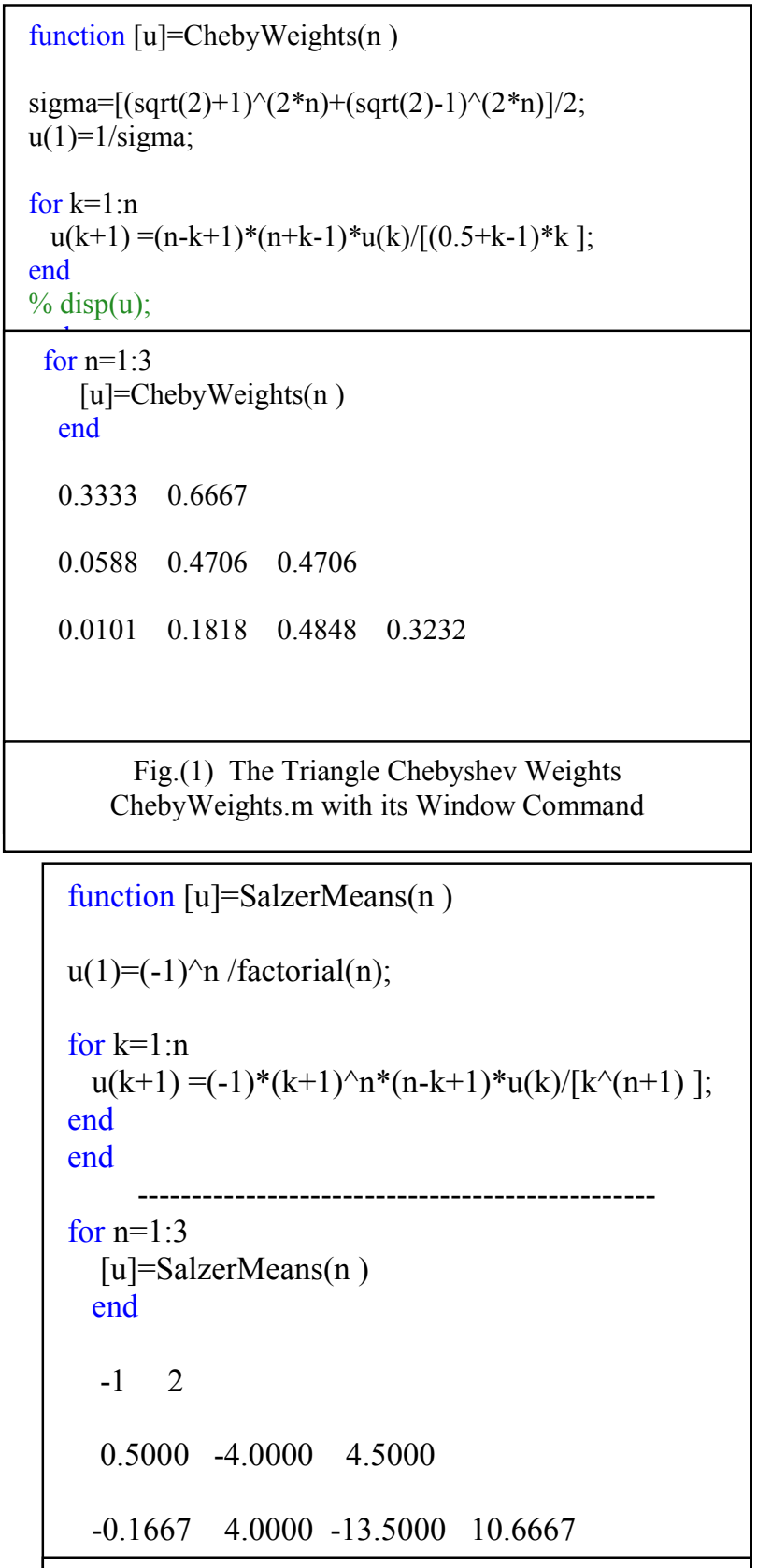

Fig.(3) The triangle Salzer Means for with its Window Command SalzerMeans.m

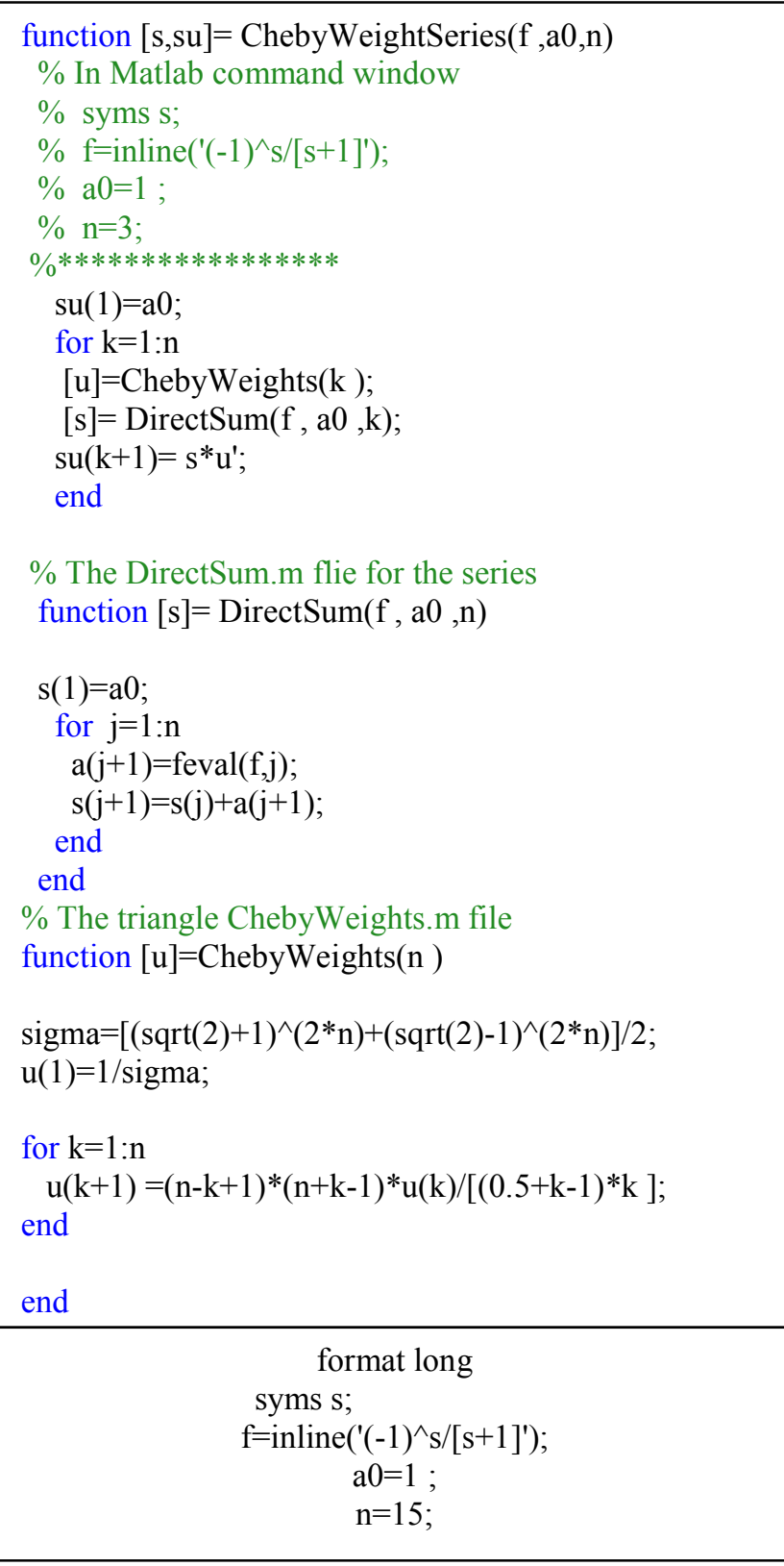

Fig.(2) The file ChebyWeightSeries.m with its Command Window for the series $\log (2)$ in Eqn.(1) The output result in Table 1 correct to 14 D.P.

series $\frac{\pi^{2}}{6}$ in Eqn.(9). The output result in Table 2 correct to 10 D.Ps

\section{EULER'S TRANSFORMATION OF SERIES}

If $\sum_{\mathrm{k}=0}^{\infty}(-1)^{\mathrm{k}} \mathrm{a}_{\mathrm{k}}=\mathrm{a}_{0}-\mathrm{a}_{1}+\mathrm{a}_{2}-\cdots$

is a convergent series with sum $s$ then

$\mathrm{S}=\sum_{\mathrm{k}=0}^{\infty} \frac{(-1)^{\mathrm{k}} \Delta^{\mathrm{k}} \mathrm{a}_{0}}{2^{\mathrm{k}+1}}$, where 


$$
\Delta^{k} a_{0}=\sum_{m=0}^{k}(-1)^{m}\left(\begin{array}{l}
k \\
m
\end{array}\right) a_{k-m}
$$

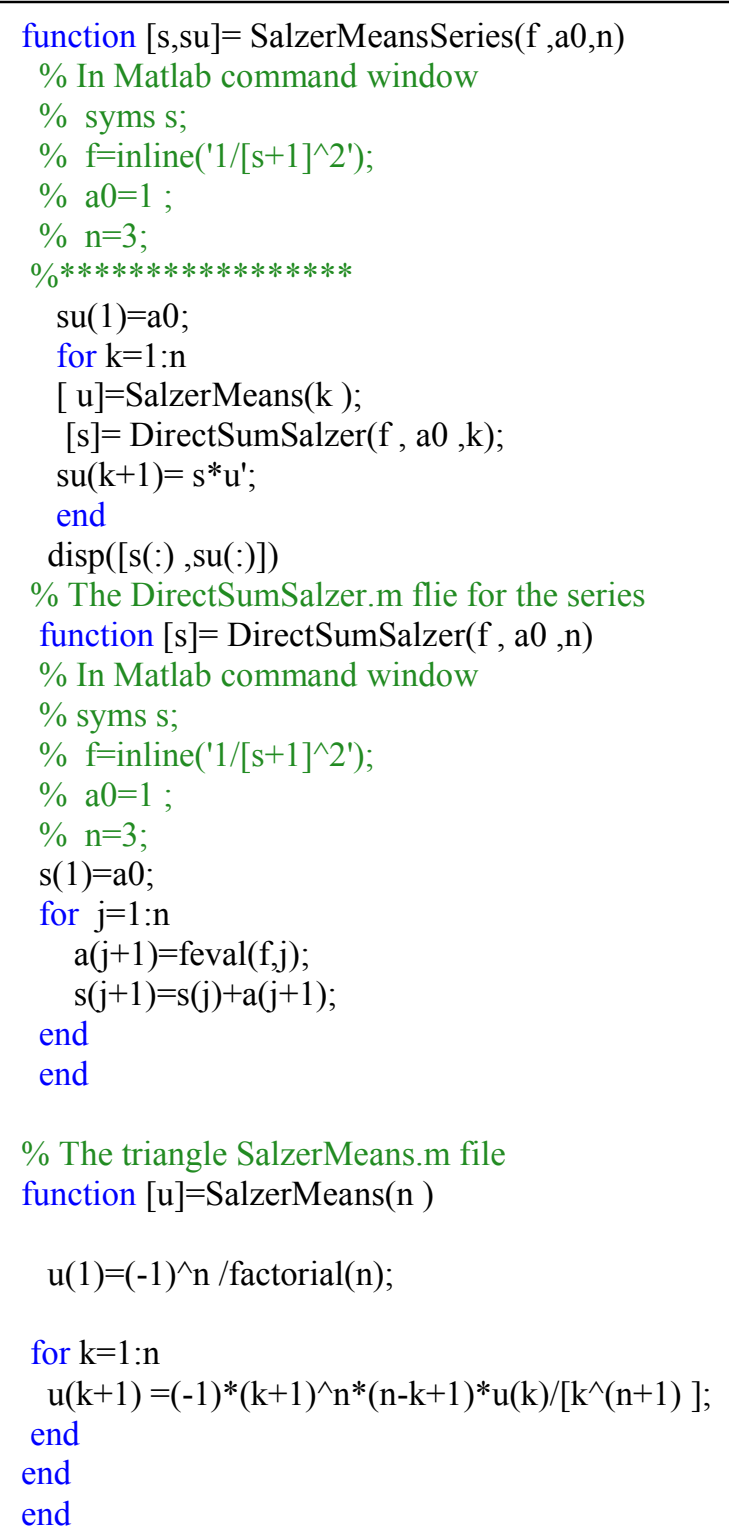

In Matlab command window syms s;

$\mathrm{f}=$ inline $\left(' 1 /[\mathrm{s}+1]^{\wedge} 2^{\prime}\right)$;

$\mathrm{a} 0=1$; $\mathrm{n}=12$;

Fig.(4) The file SalzerMeansSeries.m with its Command Window for the series $\frac{\pi^{2}}{6}$ in Eqn.(2) The output result in Table 2 correct to 10 D.P.
Example 3 Apply Euler's Transform to the series $S_{n}=\left(\ln (2)_{\mathrm{n}}=\sum_{\mathrm{k}=0}^{\mathrm{n}} \frac{(-1)^{\mathrm{k}}}{\mathrm{k}+1}(9)\right.$

The program Fig.(5) file EulerTransform.m with its CommandWindow for the series $\log (2)$ in Eqn.(9)

The output result correct to 11 D.P The sum of the series to 8 terms is 0.693147180540246 correct to 11 D.P. and with Table 3 to be followed.

\begin{tabular}{|c|c|}
\hline \multicolumn{2}{|c|}{$\begin{array}{l}\text { Table } 2 \quad \text { shows error }= \\
\frac{\pi^{2}}{6}-1.644934066775022=7.3204331 \mathrm{e}-11\end{array}$} \\
\hline $\mathrm{n}$ & su \\
\hline 1 & 1.000000000000000 \\
\hline 2 & 1.500000000000000 \\
\hline 3 & 1.625000000000000 \\
\hline 4 & 1.643518518518516 \\
\hline 5 & 1.644965277777779 \\
\hline 6 & 1.644951388888927 \\
\hline 7 & 1.644935185185290 \\
\hline 8 & 1.644933943418437 \\
\hline 9 & 1.644934041170018 \\
\hline 10 & 1.644934066247515 \\
\hline 11 & 1.644934067166105 \\
\hline 12 & 1.644934066862334 \\
\hline 13 & 1.644934066775022 \\
\hline
\end{tabular}

From Table 3 and Eqn.(12) we then obtain

$$
\begin{aligned}
& \mathrm{S}=.634524+24+\frac{.111111}{2}-\frac{(-0.11111)}{2^{2}}+\frac{.002020}{2^{3}}- \\
& \frac{(-.000505}{2^{4}}+\frac{.000156}{2^{5}} \\
& \quad=.634524+.055556+.002778+.000253+.000032+ \\
& .000005=.693148 \\
& (\mathrm{~S}=\mathrm{In} 2=.6931472 \text { to } 7 \text { D.P. })
\end{aligned}
$$

Example 4 Evaluate the integral $\int_{0}^{\infty} \frac{\sin x}{x} d x=\frac{\pi}{2}$ to 4 D.P. using the Euler transform.

$$
\begin{gathered}
\int_{0}^{\infty} \frac{\sin x}{x} \mathrm{dx}=\sum_{\mathrm{k}=0}^{\infty} \int_{\mathrm{k} \pi}^{(\mathrm{k}+1) \pi} \frac{\sin \mathrm{x}}{\mathrm{x}} d x \\
=\sum_{k=0}^{\infty} \int_{0}^{\pi} \frac{\sin (k \pi+t)}{k \pi+t} \mathrm{dt} \\
=\sum_{\mathrm{k}=0}^{\infty}(-1) \mathrm{k} \int_{0}^{\pi} \frac{\sin \mathrm{t}}{\mathrm{k} \pi+\mathrm{t}} d t
\end{gathered}
$$

Evaluating the integrals in the last sum by numerical integration we get Table (4) and from it the sum. 
The sum to $\mathrm{k}=3$ is 1.49216 . Applying the Euler transform to the remainder we obtain

$$
\begin{gathered}
\frac{1}{2}(.14180)-\frac{1}{2^{2}}(-.02587)+\frac{1}{2^{3}}(.00799) \\
-\frac{1}{2^{4}}(.00321)+\frac{1}{2^{5}}(.00153) \\
=.07090+.00647+.00100+.00020+.00005 \\
=.07862
\end{gathered}
$$

We obtain the value of the integral as $1.570 \underline{78}$ as compared with 1.57080 .

\section{Table 3 The Forward Diagonal Difference Elements by} Eqn.(12)

\begin{tabular}{|c|c|c|c|c|}
\hline \multicolumn{5}{|c|}{$\int_{0}^{\pi} \frac{\sin \mathrm{t}}{\mathrm{k} \pi+\mathrm{t}} \mathrm{dt} \mathrm{k}=0,1,2, \ldots, 8$} \\
\hline 1.85194 & & & & \\
\hline .43379 & & & & \\
\hline .25661 & & & & \\
\hline .18260 & $\Delta$ & $\Delta^{2}$ & $\Delta^{3}$ & $\Delta^{4}$ \\
\hline .14180 & & & & \\
\hline .11593 & -2587 & & & \\
\hline .09805 & -1788 & 799 & & \\
\hline .08495 & -1310 & 478 & -3211 & \\
\hline .07495 & -1000 & 310 & -168 & 156 \\
\hline
\end{tabular}

$\begin{array}{cccccc}n & u_{n} & \Delta \mathrm{u}_{\mathrm{n}} & \Delta^{2} \mathrm{u}_{\mathrm{n}} & \Delta^{3} \mathrm{u}_{\mathrm{n}} & \Delta^{3} \mathrm{u}_{\mathrm{n}} \\ 9 & .111111 & & & & \\ 10 & .100000 & -11111 & & & \\ 11 & .090909 & -9091 & 2020 & & \\ & & & & & \\ 12 & .083333 & -7576 & 1515 & -505 \\ 13 & .007692 & -6410 & 1166 & -349 & 156\end{array}$

Table 4 Gaussian Quadrature 5-points to evaluate $\int_{0}^{\pi} \frac{\sin \mathrm{t}}{\mathrm{k} \pi+\mathrm{t}} \begin{gathered}\text { and the Diagonal elements by Eqn. (12) } \\ \text { and }\end{gathered}$

\section{In Example 4}

$$
\int_{0}^{\pi} \frac{\sin \mathrm{t}}{\mathrm{k} \pi+\mathrm{t}} \text { using quassianTableInf. m Fig. (6) }
$$

and the Diagonal elements by Eqn. (12) the value of integral $\int_{0}^{\infty} \frac{\sin x}{x} \mathrm{dx}$ is computed accurately to 5 D.P. for $\mathrm{k}=8$; in the command window in Fig.(6) Howeverthe result is 1.570796318151585 when used only 5 point quadrature Gaussian Rule and so to get more accuracy one needs to use higher points in the rule.
This can be done by sight change in the file quassian Table In f.m ,where in the command window let $\mathrm{k}=18$; to get the result accurately to 9 D.P. which is equal 1.570796318151585 and $\mathrm{pi} / 2-1.570796318151585=8.643311 \mathrm{e}-09$

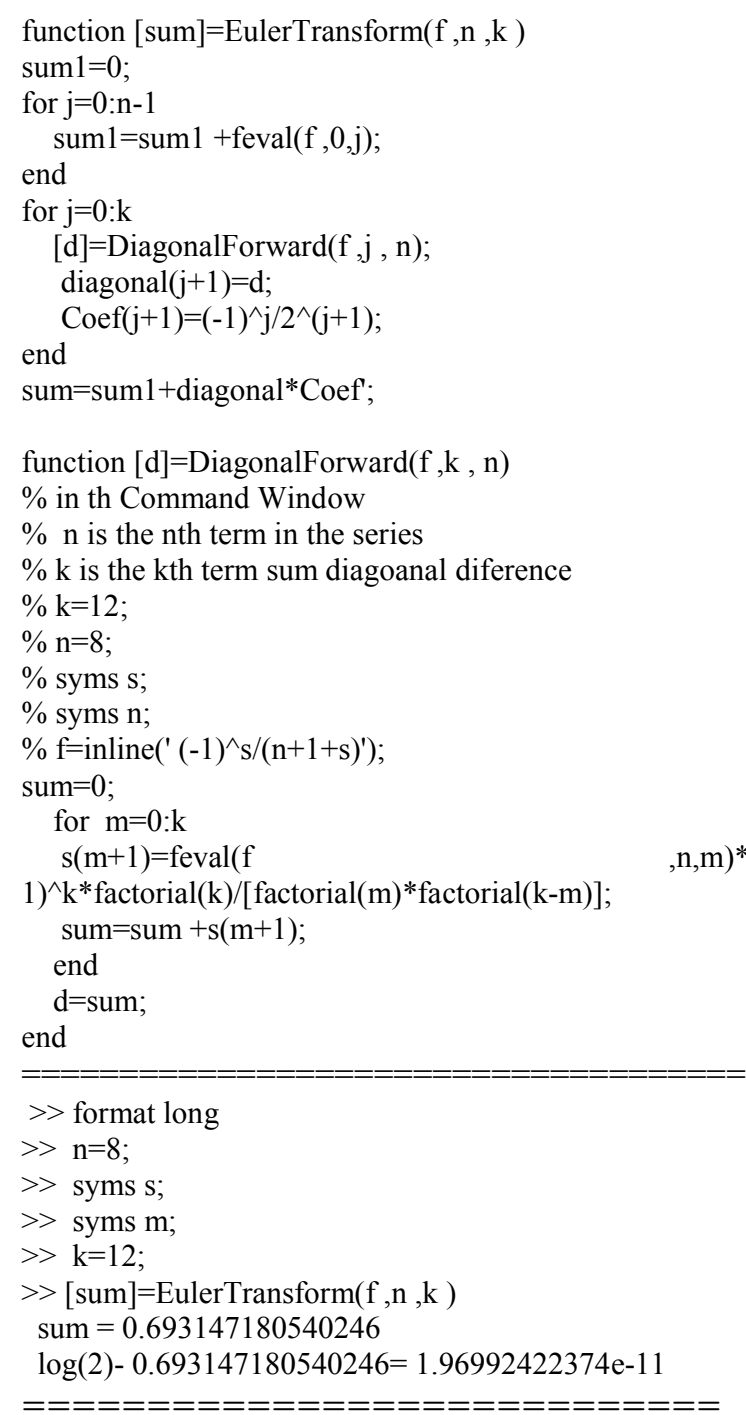

Fig.(5) The file EulerTransform.m with its Command Window for the series $\log (2)$ in Eqn.(9). The output result correct to 11 D.P 
easily. The table 3 and Table 4 can be displayed easily from the file ForwadDifrenceTable.m or DiagonalForward.m ((but one require a little adjustment to employ an array variables instead of sum)). In ,Example 5 below We will describe another important Euler's formula

called

Euler - Maclaurin summation formula

$$
\begin{aligned}
& \text { 4.EULER-MALCAURIN SUMMATION } \\
& \text { FORMULA } \\
& \qquad \begin{array}{c}
\sum_{k=1}^{n-1} f_{k}=\int_{0}^{n} \int(k) d k-\frac{1}{2}[f(0)+f(n)]+\frac{1}{12}\left[f^{\prime}(n)-\right. \\
\left.f^{\prime(0)}\right]-\frac{1}{720}\left[f^{\prime \prime \prime}(n)-f^{\prime \prime \prime}(n)\right] \\
\quad+\frac{1}{30240}\left[\mathrm{f}^{(\mathrm{V})}(\mathrm{n})-\mathrm{f}^{(\mathrm{V})}(0)\right] \\
-\frac{1}{1209600}\left[f^{(V I I)}(n)-f^{(V I I)}(0)\right]+\cdots(14)
\end{array}
\end{aligned}
$$

Example 5.

Sum the series $\sum_{\mathrm{k}=1}^{\infty} \mathrm{k}^{-2}=\frac{\pi^{2}}{6}$ using

the Euler - Maclaurin summation formula.

$$
\begin{gathered}
\sum_{k=1}^{\infty} \mathrm{k}^{-2}=\sum_{k=1}^{10} \mathrm{k}^{-2}+\sum_{k=1}^{\infty}(k+10)^{-2} \\
=\sum_{\mathrm{k}=1}^{10} \mathrm{k}^{-2}+\int_{0}^{\infty} f(k) d k-\frac{1}{2} \mathrm{f}_{0}-\frac{1}{12} \mathrm{f}_{0}^{\prime}+\frac{1}{720} \mathrm{f}^{\prime \prime \prime}{ }_{0} \\
-\cdots \quad(14) \\
\sum_{\mathrm{k}=1}^{\infty} \mathrm{k}^{-2}=1.549767731+.1-.005 \\
+.000166667-.000000333 \\
=1.644934056
\end{gathered}
$$

as compared with $\frac{\pi^{2}}{6}=1.644934067$.

The important about this formula in Eqn.(14) is that it combines both the methods for either evaluating an infinite series or an infinite integrals whenever the other one is known or it can be computed. While the reader must observe that there was a restriction for evaluating the first , the second , the third derivatives and so fourth ,etc., it can't be considered as a disappointed to the method for it can give a rough estimate if evaluating either one of them easily. This clearly seen here for the integral

$$
\int_{0}^{\infty}(\mathrm{k}+10)^{-2} \mathrm{dk} \text { in Eqn. (14) }
$$

Alternatively , the the general Euler - Maclaurin summation formula is given $\sum_{k=a}^{\infty} f_{k}=\int_{a}^{\infty} f(x) d x+\frac{1}{2} f(a)$

$$
\begin{aligned}
& -\sum_{\ell=2}^{k} \frac{(-1)^{\ell}}{\ell !} f^{(\ell-1)}(a) B_{\ell} \\
& -\frac{(-1)^{k}}{k !} \int_{a}^{\infty} f^{(k)}(x) \psi_{k}(x) d x
\end{aligned}
$$

Where $B_{\ell}$ is Bernoulli numbers and

$$
\psi_{k}(x)=B_{\ell} \text { are the Bernoulli polynomials }
$$

Despite the fact that in Example 5 the sum of the series $\sum_{\mathrm{k}=1}^{\infty} \mathrm{k}^{-2}=\frac{\pi^{2}}{6}$ is computed to 8 D.P. as We cited from [ 3 ], We give another $\quad$ Example 6 showing Euler - Maclaurin summation formula. is not recommended in general to evaluate an infinite integral

Example 6 Evaluate the incomplete gamma function $\mathrm{T}\left(-\alpha, \frac{-1}{z}\right)$ when $\alpha=0$ and $z=-1$

i.e. evaluate the integral $\int_{0}^{\infty} \frac{e^{-x}}{1+x} d x$. Now apply Eqn.(14) as to get

$$
\begin{gathered}
\sum_{k=0}^{\infty} \frac{e^{-x}}{1+x}=\int_{0}^{\infty} \frac{e^{-x}}{1+x} d x+\frac{1}{2} f(0)+\frac{1}{12}[-2] \\
+\frac{1}{720}[16]+\frac{1}{30240}[-326] \\
-\frac{1}{1209600}[-13700]+\cdots(16)
\end{gathered}
$$

Hence ,to evaluate $\int_{0}^{\infty} \frac{e^{-x}}{1+x} d x$ in Eqn. (16), We

need

to

evaluate

$\sum_{k=1}^{\infty} \frac{e^{-x}}{1+x}$ by using Levin'straform as in Fig. (7)

The result for this series is computed accurately to

0.283489078864866 to 12 D.P. From Eqn.(16), We

get $\int_{0}^{\infty} \frac{e^{-x}}{1+x} d x=0.6396$ which is incorrect

showing that in general Euler - Maclaurin summation formula must be avoided to evaluate an infinite integral.

\section{EVALUATION ON INFINITE INTEGRALS}

As ,We have seen ,above both Euler's Transformations and the rest of linear transformation $s$ mentioned can be used to evaluate an infinite whenever it can expressed as a series .Further one of fundamentals series is Levin's Transform that discussed in [ 2 ] , and [12 ]. Here , We applied to sum the series $\sum_{k=1}^{\infty} \frac{e^{-x}}{1+x}$ by Fig. (7)

in the APPENDIX using Levin's Transform.

\subsection{Levin's Transform}

Levin's Transform is on e of the robot fundamental

Transformations that compute most of the series expansions. In [2 ] , we have been shown that it can compute to more than 16 decimal places (D.P.) of accuracy using FORTRAN LANGUAGE with the FULL MACHINE ACCURACY. Unfortunately the program is not there but only a computational remarks in [12] 
expressing how to use the command to obtain the full machine accuracy whenever the FUNCTION LEVIN PRGRAM is called and expressed .

Fully when it is called.For such a reason the program is so long it can't followed to be practical.

However, when applied using FORMAT LONG with a MATALA PROGRAM it calculates similarly as in FORTAN LANGUAGE using full machine accuracy. Further, unfortunately,Levin's transform breaks down to fail for very slowly convergent or divergent series as can be seen in [ 12 ]. Here, We applied to compute $\int_{0}^{\infty} \frac{e^{-x}}{1+x} d x$ as in Example 7

Example 7 compute $\int_{0}^{\infty} \frac{e^{-x}}{1+x} d x$ using Levin's

Transform to evaluate it

$$
\int_{0}^{\infty} \frac{e^{-x}}{1+x} \mathrm{dx}=\sum_{\mathrm{k}=0}^{\infty} \int_{\mathrm{k}}^{(\mathrm{k}+1)} \frac{e^{-x}}{1+x} d x
$$

The result $\int_{0}^{\infty} \frac{e^{-x}}{1+x} \mathrm{dx}=0.5963473$ accurate to 7 D.P. of accuracy as can be shown in Table 5 using only 10 terms while direct summation uses 20 terms .

Alternatively , apply partial integration the integral in Eqn.(17) can be expressed as in Eqn.(18)

$$
\begin{aligned}
\mathrm{I}_{\mathrm{x}}= & \int_{x}^{\infty} \frac{e^{-t}}{t} \mathrm{dt}=\frac{e^{-x}}{x}\left[1-\frac{1 !}{\mathrm{x}}+\frac{2 !}{\mathrm{x}^{2}}-\frac{3 !}{\mathrm{x}^{3}}+\cdots\right. \\
& \left.+(-1)^{(\mathrm{n}-1)} \frac{(\mathrm{n}-1) !}{\mathrm{x}^{\mathrm{n}-1}}\right]+R_{n}(18) \\
& \text { where } R_{n}=(-1)^{n} \cdot n ! \cdot \int_{x}^{\infty} \frac{e^{-t}}{t} d t
\end{aligned}
$$

Thus, We have $\left|R_{n}\right|<e^{-x} n ! / x^{n+1}$ and by definition

$$
\frac{e^{-x}}{x}\left[1-\frac{1 !}{\mathrm{x}}+\frac{2 !}{\mathrm{x}^{2}}-\frac{3 !}{\mathrm{x}^{3}}+. .+(-1)^{(\mathrm{n}-1)} \frac{(\mathrm{n}-1) !}{\mathrm{x}^{\mathrm{n}-1}} \ldots\right]
$$

Hence, for large values of $\mathrm{x}$ the terms first decrease in absolute value but diverge when $n \rightarrow \infty$, since $x$ is fixed . Now if $x=10$ and one uses direct summation to get

$$
\mathrm{I}_{\mathrm{x}}=\int_{x}^{\infty} \frac{e^{-t}}{t} \mathrm{dt}=e^{-10} *(0.0915633)
$$

\subsection{Transform to Continued Fraction Technique}

Since, as we have been shown in [6] series expansions cab be transformed to a continued fractions, we first expand the required integral

$$
\mathrm{I}_{\mathrm{x}}=\int_{x}^{\infty} \frac{e^{-t}}{1+t} \mathrm{dt} \quad x=0,1,2,3, . .
$$

to $\mathrm{I}_{\mathrm{x}}=\frac{e^{-x}}{1+x}\left[1-\frac{1 !}{1+\mathrm{x}}+\frac{2 !}{(1+\mathrm{x})^{2}}-\frac{3 !}{(1+\mathrm{x})^{3}}+\right.$

$$
\left.\ldots+(-1)^{(\mathrm{n}-1)} \frac{(\mathrm{n}-1) !}{(1+\mathrm{x})^{\mathrm{n}-1}} . .\right]
$$

$$
\int_{0}^{\infty} \frac{e^{-t}}{x+t} d t=\frac{1}{x+1} \frac{1}{x+1} \frac{1}{1+x+} \frac{2}{1+x+\ldots} \frac{3}{x}
$$

or as

$$
\begin{aligned}
& e^{-x} \int_{\mathrm{x}}^{\infty} \frac{\mathrm{e}^{-\mathrm{t}}}{\mathrm{t}} \mathrm{dt} \\
& =\frac{1}{\mathrm{x}+1-\mathrm{x}+3-\mathrm{x}+5-\mathrm{x}+7-\mathrm{x}+9} \frac{1}{\cdots}
\end{aligned}
$$

By evaluating Eqn.(21) and Eqn.(22), We deduce that there are many so approaches can be used to evaluate infinite integrals or incomplete an infinite integral.

\section{COMPUTATIONAL REMARKS}

When writing a program for any one for the linear transformations Cheyshev or Salzer or similar

transformations, one must check that the sum of absolute value elements of triangles u's in any row

direction is equal to one before applying the series terms to find its sum.

When evaluating an infinite integral by expressing it as a series summations values of integrals, then the ranges of these integrals must be very small as in Eqn.(17) or even be more smaller as $\left[\mathrm{k}^{*} 0.5,(\mathrm{k}+1) * 0.5\right]$ for $\mathrm{k}=0,1,2, \ldots$ with Gaussian qaudrature rule one must use at least 10 points to get more accuracy rather than, We have used 5 points .

Applying integration by parts the infinite integral

$$
\int_{0}^{\infty} \frac{e^{-x}}{1+x} d x=\sum_{n=0}^{\infty}(-1)^{n} n !
$$

Eqn.(23) is an asymptotic divergent which can be sum with other alterative methods that may be ,We will be discussed them in the futures.

and then in general to C.F. as 
Some Linear Transformations with MATLAB Applications, Especially On Infinite Integrals

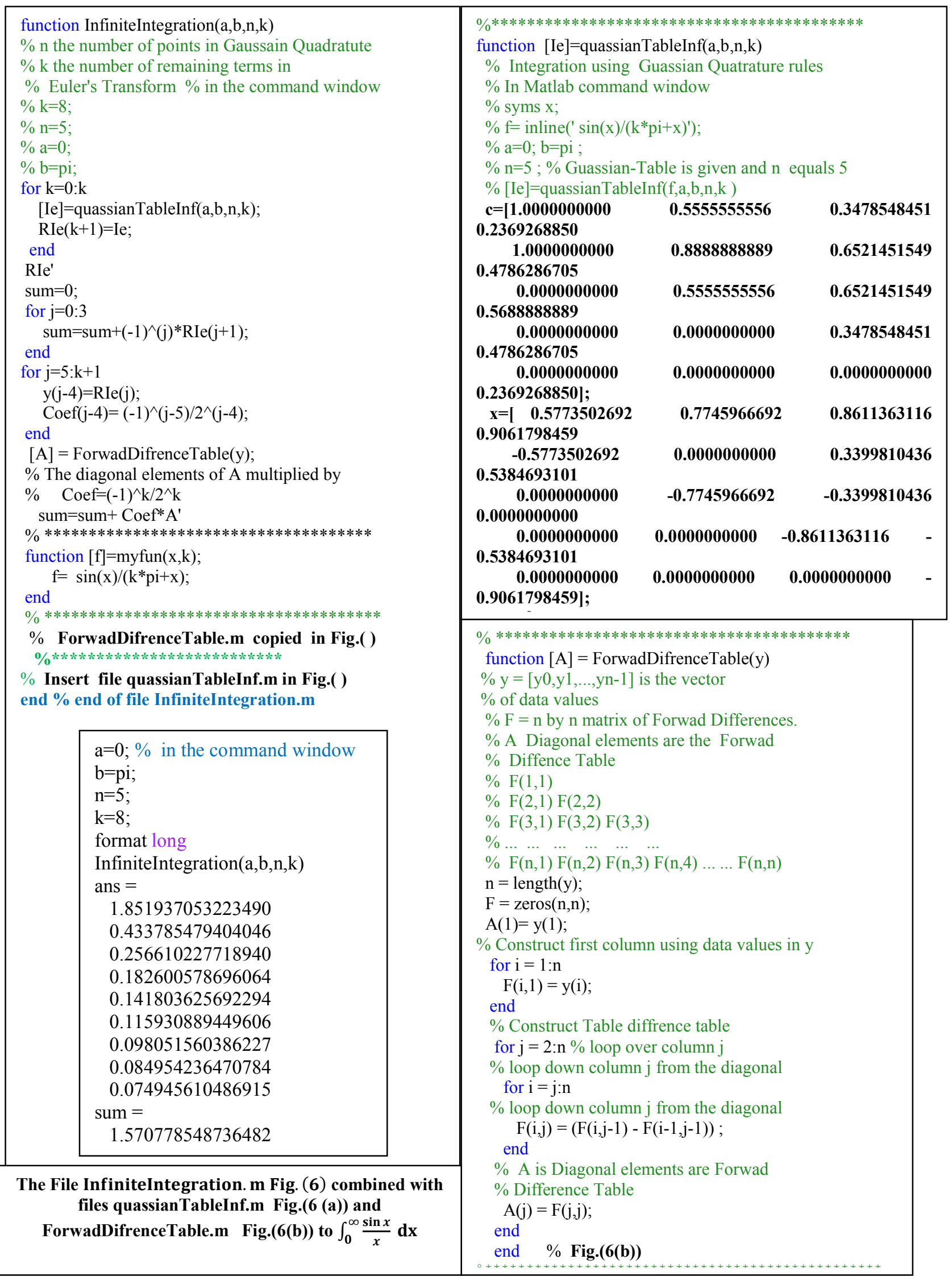


Table 5 Gaussian Quadrature 5-points to evaluate $I_{k}=\int_{\mathrm{k}}^{(\mathrm{k}+1)} \frac{e^{-x}}{1+x}$ $\mathrm{k}=0,1,2, \ldots . .20$ using Direct Sum $S_{k} \&$ Levin's Sum $U_{(2 k+2)}$

\begin{tabular}{|c|l|c|c|}
\hline $\mathrm{k}$ & $I_{k}$ & & \\
\hline 0 & 0.463421930906830 & 0.463421930906830 & 0.593821335725283 \\
1 & 0.097456192252491 & 0.560878123159321 & 0.596341511720131 \\
2 & 0.025195832234349 & 0.586073955393670 & 0.596347295689531 \\
3 & 0.007151953938887 & 0.593225909332557 & 0.596347300371702 \\
4 & 0.002142585452849 & 0.595368494785406 & \\
5 & 0.000664893693863 & 0.596033388479269 & Levin's Sum uses only \\
6 & 0.000211526114408 & 0.596244914593677 & $\left.2^{*} \mathrm{k}+2\right)=10$ \\
7 & 0.000068550361456 & 0.596313464955133 & Terms . The result is \\
8 & 0.000022535603570 & 0.596336000558703 & 0.596347300371702 \\
9 & 0.000007493402231 & 0.596343493960934 & accurate to $7^{\text {th }}$ decimal \\
10 & 0.000002514932932 & 0.596346008893866 & places \\
11 & 0.000000850608992 & 0.596346859502858 & \\
12 & 0.000000289579408 & 0.596347149082266 & \\
13 & 0.000000099135826 & 0.596347248218092 & \\
14 & 0.000000034102998 & 0.596347282321089 & \\
15 & 0.000000011781184 & 0.596347294102273 & \\
16 & 0.000000004085096 & 0.596347298187369 & \\
17 & 0.000000001421189 & 0.596347299608558 & \\
18 & 0.000000000495890 & 0.596347300104448 & \\
19 & 0.000000000173490 & 0.596347300277937 & \\
& & & \\
& & & \\
\hline
\end{tabular}




\section{ACKNOWLEDGEMENT}

I would thank the International Centre of Theoretical Physics(ICTP), ITALY for granting me a junior associate membership during 1988-1992

to send me both the references [ 4 ] and [5 ]due to an ARAB GRANT . However, I do BLAME the ARAB for they didn't follow it to be SEPENT all of it . In FACT, I have GOT LITTLE of it .

Second, I am gratefully of thanks to people, Government of SUDAN and University of Wales, UNITED KINDOM to get my M.Sc. and Ph.D. and for which I have collect the precious reference [2].

\section{REFERENCES}

[1] Dafalla Awadalla Gismalla , Numerical Methods and Algorithms using Matlab Programming Language, LAMBERT Academic Publishing https://www.lap-publishing.com/site/howto-publish $/ 14$

[2] D.A.Gismalla ,et al .Acceleration of Convergence of Series for Certain Multiple Integrals. International Journal of Computing Mathematics I. J.C.M, Vol. 24, pp 55-68, 1987.

[3] Abramowitz,M , and Stegun ,I.A. 1964 ,Handbook of Mathematical Functions, Applied Mathematics Series, Volum 55 (Washington : National Bureau of Standards; reprinted 1968 by Dover Publication New York )

[4] Clark-Erik Frberg, Numerical Mathematics, Theory and Computer, Application. The Benjamin/Cummings Publishing Company, Inc , 1985

[5] Jet Wimp, Sequence Transformations and Their applications , Mathematics in Sciences and Engineering, Vol.154, 1981 ,Academic Press, London, New York.
[6] D.A. Gismalla, Survey on Transformations for Infinite Series to Continued Fractions with MATLAB Program for Computing Some, International Journal of Innovative Research in Engineering \& Management(IJIERM) ISSN:2350-0557,Volume 3 , Issue 3 , May 2016 http://www.ijirem.org/IJIREM/Arch.aspx?rep=12

[7] D.A.Gismalla On Evaluations Some Special Functions with Pade Approximant in Mupad Interface and Mathematica , International Journal Engineering Research \& Management(IJERM) ISSN:2349-2058, Volume 3 ,Issue 5 ,May 2016 https://www.ijerm.com/vol/Volume-03Issue-05

[8] D.A.Gismalla \& Mohamed H. A. ELHEBIR,Factorizations , Multiplications and Views on Parallel Computing. International Journal of Innovative Research in Engineering \& Management (IJIREM) ISSN: 2350-0557, Volume-3, Issue-6, November-2016 http://www.ijirem.org/IJIREM/Arch.aspx?rep=15

[9] Petersen,G.M. (1966). "Regular Matrix Transformations". McGraw-Hill ,New York.

[10] Peyerimhoff.A. (1969)." Lecture Notes of Summability" . Lecture Notes In Mathematics

[11] Hardy ,G.H.(1956). " Divergent Series." Oxford Univ. Press , London and

[12] Methods for the Evaluations of N-dimensional Integrals, (a Thesis for the Ph.D. , Software was written using FORTRAN Language with Full Machine Accuracy), University of Wales, U.K. 1984 http://www.philadelphia.edu.jo/newlibrary/thesisdissertation/406-mathematics/25864-4382 


\section{APPENDIX}

function LevinTranfSum3(n,a0)

\% Integration using Levin Transform for Triple Integration on cubic.

$\%$ This Technique is a series Technique for which the integral is expressed

$\%$ as a series FIRST and then LEVIN is applied. The general term for the

$\%$ series can be expressed as an inline function or a handle object with

$\%$ the initial term submitted to the program in advanced to generate the

$\%$ other terms with the number of terms $n$ to be taken for the sum.

$\%$ The function $\mathrm{f}$ in LevinTranfSum $(\mathrm{f}, \mathrm{n}, \mathrm{a} 0)$ is a ratio to generate other terms .

$\%$ In Matlab command window

$\% \mathrm{a} 0=\exp (-1) / 2$;

$\% \mathrm{n}=20$;

$\%$ LevinTranfSum $(\mathrm{n}, \mathrm{a} 0)$

global UT ;

for $\mathrm{k}=1: \mathrm{n}$

$[\mathrm{S}, \mathrm{UT}]=$ LevinTransform $(\mathrm{k}, \mathrm{a} 0)$; $\mathrm{F}(\mathrm{k})=\mathrm{UT}$;

format long

$\mathrm{n}=20$;

$\mathrm{a} 0=\exp (-1) / 2$;

LevinTranfSum $3(\mathrm{n}, \mathrm{a} 0)$

Fig.(7) is LevinTranfSum3.m to sum the series $\sum_{k=1}^{\infty} \frac{e^{-k}}{k+1}=0.283489078864866$ to 12 D.P. end

$\operatorname{disp}($ ' The Sum of the Series having $2 * \mathrm{n}+2$ terms')

$\operatorname{disp}\left(\left[\mathrm{S}^{\prime}\right]\right)$;

$\operatorname{disp}($ ' The Sum of the Series using LEVIN TRANSFORM using $2 * n+2$ terms')

$\operatorname{disp}\left(\left[F^{\prime}\right]\right)$;

function $[\mathrm{S}, \mathrm{UT}]=$ LevinTransform $(\mathrm{k}, \mathrm{a} 0)$

$\mathrm{a}(1)=\mathrm{a} 0$;

$\mathrm{S}(1)=\mathrm{a}(1)$;

$\mathrm{C}(1)=1$;

TotalSumDen $(1)=1$;

TotalSumNum $(1)=1$;

for $\mathrm{j}=1: 2 * \mathrm{k}+1$

$\mathrm{a}(\mathrm{j}+1)=\exp (-1) *(1+\mathrm{k}) /(2+\mathrm{k}) * \mathrm{a}(\mathrm{j})$;

$\mathrm{S}(\mathrm{j}+1)=\mathrm{S}(\mathrm{j})+\mathrm{a}(\mathrm{j}+1)$;

$\mathrm{C}(\mathrm{j}+1)=(2 * \mathrm{k}+2-\mathrm{j}) * \mathrm{C}(\mathrm{j}) /(\mathrm{j})$;

TotalSumDen $(\mathrm{j}+1)=$ TotalSumDen $(\mathrm{j})+(-1)^{\wedge} \mathrm{j} * \mathrm{C}(\mathrm{j}+1) *(\mathrm{j}+1)^{\wedge}(2 * \mathrm{k}-1) * \mathrm{~S}(\mathrm{j}+1) / \mathrm{a}(\mathrm{j}+1)$;

TotalSumNum $(j+1)=$ TotalSumNum $(j)+(-1)^{\wedge} j^{*} C(j+1)^{*}(j+1)^{\wedge}(2 * k-1) / a(j+1)$; end

$\mathrm{UT}=\operatorname{TotalSumDen}(2 * \mathrm{k}+2) / \operatorname{TotalSumNum}(2 * \mathrm{k}+2)$; 